\title{
Serum soluble Klotho protein level is associated with residual diuresis in incident peritoneal dialysis patients
}

\author{
Edyta Golembiewska ${ }^{\circledR}$, Krzysztof Safranow², Joanna Kabat-Koperska', Marek Myślak and \\ Kazimierz Ciechanowski ${ }^{1}$
}

1Department of Nephrology, Transplantology and Internal Medicine, Pomeranian Medical University, Szczecin, Poland; ${ }^{2}$ Department of Biochemistry and Medical Chemistry, Pomeranian Medical University, Szczecin, Poland

\begin{abstract}
Aim: Active vitamin D (1,25-dihydroxyvitamin $\left.\mathrm{D}_{3}\right), \mathrm{PTH}$, fibroblast growth factor-23 (FGF-23) and Klotho protein are key regulators of phosphate metabolism. Hyperphosphatemia and increased FGF-23 level in patients with end-stage renal disease are associated with increased morbidity and mortality. The relationships among key regulators of phosphate metabolism are still being investigated. FGF-23, the humoral factor involved in phosphate metabolism, is strongly associated with serum phosphorus level. Klotho, a transmembrane protein expressed primarily in renal tubules, functions as an obligatory co-receptor for FGF-23. The soluble form of Klotho, produced by the shedding of the transmembrane protein, is detectable in body fluids. The purpose of the study was to assess if serum soluble alpha-Klotho level was related to phosphate metabolism parameters and residual renal function (RRF) in incident peritoneal dialysis (PD) patients. Methods: Thirty-five clinically stable patients 4 to 6 weeks after the onset of PD were included in the study. For each patient, clinical and laboratory data were reviewed. Serum phosphorus concentration, urinary and peritoneal phosphate clearance, serum FGF-23 and soluble Klotho protein concentrations were determined. Results: Serum soluble alpha-Klotho was strongly negatively correlated with 24-hour diuresis $(R s=-0.55, p=0.004)$ and renal phosphate clearance (Rs $=-0.40, p=0.049$ ), but not with RRF. Conclusions: Serum soluble Klotho protein concentration is inversely related to residual diuresis and renal phosphate clearance in incident PD patients.
\end{abstract}

Key words: FGF-23, peritoneal dialysis, phosphate, residual diuresis, soluble Klotho

Received: 30 December, 2012; revised: 03 April, 2013; accepted: 23 May, 2013; available on-line: 07 June, 2013

\section{INTRODUCTION}

The identification of Klotho gene in 1997 by Kuroo and colleagues (Kuro-o et al., 1997) was a major discovery as the gene encodes a protein regulating multiple functions. A defect in Klotho gene expression in mice results in a phenotype of premature aging including shortened life span, growth retardation, hypogonadism, skin and muscle atrophies, vascular calcification, cognition impairment, motor neuron degeneration and others. This aging-like phenotype is associated with phosphate balance disorders and underlines the major function of Klotho in mineral metabolism (Torres et al., 2007; Wang \& Sun, 2009). As another Klotho gene was identified en- coding a protein expressed in adipose tissue, liver and pancreas (being a co-receptor for FGF15/19 and involved in bile acid and energy metabolism), it was named beta-Klotho. This discovery also led to the revised naming of Klotho as alpha-Klotho (Hu et al., 2012; Martin et al., 2012). Alpha-Klotho is predominantly expressed in the kidneys and choroid plexus in the brain, low expression is also reported in the pituitary gland, placenta, skeletal muscle, urinary bladder, aorta, pancreas, ovary, testis and colon (Martin et al., 2012). Two forms of alpha-Klotho protein have been reported: a membrane and a secreted one. Membrane Klotho forms a complex with fibroblast growth factor (FGF) receptors and functions as an obligate co-receptor for the FGF-23 phosphatonin in distal tubules. The secreted form of Klotho seems to function as a humoral factor and regulates glycoproteins on the cell surface including ion channels and growth factors (Cheng et al., 2011; Drueke \& Prie, 2007; Razzaque, 2009). There are data suggesting that secreted Klotho exerts phosphaturic effects independently of FGF-23. Studies in mice showed that intravenously administered soluble Klotho induced phosphaturia both in normal and FGF-23 null mice and inactivated NaPi2a proximal tubule transporter. Soluble Klotho has also been detected in the luminal fluid of proximal tubules, but how it gets there is not known (Hu et al., 2010). The exact functions of soluble Klotho are yet to be defined. Circulating soluble Klotho is produced either by proteolytic cleavage of the extracellular domain of the transmembrane form (130 kDa isoform) by two membraneanchored proteases (ADAM10 and ADAM17) or by alternative mRNA splicing (70 $\mathrm{kDa}$ isoform) (Huang \& Moe, 2011). There is a paucity of data on levels of soluble alpha-Klotho in patients with chronic kidney disease (CKD) and/or undergoing dialysis.

The aim of this study was to assess if serum and dialysate soluble Klotho levels could be associated with parameters of residual renal function and of phosphate metabolism, including FGF-23, at the onset of peritoneal dialysis (PD).

\section{SUBJECTS AND METHODS}

Patients. Between January 2008 and November 2011, thirty-five incident peritoneal dialysis patients at the Peritoneal Dialysis Center, Department of Ne-

e-mail: irys@pum.edu.pl

Abbreviations: CKD, chronic kidney disease; ESRD, end-stage renal disease, FGF-23, fibroblast growth factor-23; PD, peritoneal dialysis; $\mathrm{PTH}$, parathyroid hormone; RRF, residual renal function 
phrology, Transplantology and Internal Medicine, Szczecin, Poland, were enrolled in the study 4-6 weeks after the dialysis onset. All patients were started on continuous ambulatory peritoneal dialysis (CAPD) using four $2 \mathrm{~L}$ glucose-based dialysates with calcium concentration of $1.25 \mathrm{mmol} / \mathrm{L}$ (Baxter Healthcare). The patients were clinically stable. None had peritonitis at the time of the study or in the 4 weeks preceding the study. Twenty-five patients used calcium-based phosphate binders and alphacalcidol. None of the patients received cinacalcet.

All patients gave written informed consent. The study has been approved by the local Bioethics Committee.

The charts of these patients were reviewed to assess age, gender, body surface area (BSA), type of nephropathy, presence of diabetes and residual renal function (RRF).

The main characteristics of the study group are presented in Table 1 . The causes of end-stage renal disease (ESRD) were as follows: diabetes (3), glomerulonephritis (11), hypertension (4), chronic pyelonephritis (2), other (7), unknown (8). The other causes of ESRD were: obstructive nephropathy (2), systemic lupus erythematosus (2), rheumatoid arthritis (1), multiple myeloma (1), chemotherapy complication (1). In the patient with chronic kidney disease due to chemotherapy complication the treatment of the underlying disease had been terminated more than three years earlier and there were no signs of recurrence of the disease. Patients with SLE and rheumatoid arthritis were in clinical and laboratory remission.

Measurements of serum urea, creatinine, calcium, phosphate, iPTH, albumin and CRP were performed using routine laboratory techniques on a Cobas Integra 800 device, Roche.

Urine and dialysate specimens were collected over 24 hours to determine urinary and peritoneal phosphate clearance (using UV/P formula). The patients were able accurately to carry out urine collection and peritoneal dialysis exchanges.

Table 1. Characteristics of study group.

\begin{tabular}{ll}
\hline Age (years) & $46.1 \pm 16.1$ \\
Sex (males/females) (\%) & $18 / 17(51 \% / 49 \%)$ \\
Diabetes (\%) & $3(8.6 \%)$ \\
RRF (residual renal function) $\left(\mathrm{mL} / \mathrm{min} / 1.73 \mathrm{~m}^{2}\right)$ & $10.2 \pm 4.8$ \\
Diuresis (mL/day) & $2041 \pm 1067$ \\
Renal phosphate clearance (L/week) & $63.64 \pm 44.74$ \\
Peritoneal phosphate clearance (L/week) & $35.36 \pm 9.6$ \\
Kt/V & $3.23 \pm 1.05$ \\
D/P crea & $0.65 \pm 0.10$ \\
Serum albumin (g/L) & $38.4 \pm 4.38$ \\
Serum C-reactive protein (mg/L) & $10.4 \pm 16.2$ \\
Serum urea (mg/dL) & $89.9 \pm 29.9$ \\
Serum creatinine (mg/dL) & $5.2 \pm 2.4$ \\
Serum calcium (mmol/L) & $2.2 \pm 0.2$ \\
Serum phosphorus (mmol/L) & $1.4 \pm 0.4$ \\
Serum iPTH (pg/mL) & $483 \pm 348$ \\
Serum FGF-23 (pg/mL) & $557 \pm 856$ \\
Serum soluble alpha-Klotho protein $(\mathrm{pg} / \mathrm{mL})$ & $165.9 \pm 90.2$ \\
Dialysate soluble alpha-Klotho protein $(\mathrm{pg} / \mathrm{mL})$ & $9.9 \pm 8.7$ \\
\hline
\end{tabular}

$\mathrm{D} / \mathrm{P}$ crea, dialysate to plasma ratio for creatinine in PET test; iPTH, intact parathyroid hormone; FGF-23, fibroblast growth factor -23 ; RRF, residual renal functions evaluated using creatinine clearance determined by the UV/P formula: RRF $=24-h$ urine creatinine concentration $(\mathrm{mg} / \mathrm{mL}) \times 24$-h urine output $(\mathrm{mL}) /$ serum creatinine $(\mathrm{mg} / \mathrm{mL}) \times 1440$. Data are given as mean \pm S.D. or number (percent).
Serum FGF-23 levels were determined with a sandwich enzyme-linked immunosorbent assay (Human FGF23 ELISA Kit, Millipore). Serum and dialysate soluble Klotho levels were determined using a solid phase sandwich ELISA using two kinds of highly specific antibodies (soluble alpha-Klotho human ELISA, Demeditec).

Statistical analysis. In patients with undetectable Klotho in dialysate, for statistical purposes, the value of Klotho concentration was set at 0 . Spearman's rank correlation coefficient (Rs) was used to measure associations between quantitative variables. Mann-Whitney test was used to compare values between groups. $P$-value $<0.05$ was considered statistically significant.

\section{RESULTS}

Mean serum and dialysate soluble alpha-Klotho levels are shown in Table 1. Serum Klotho and FGF23 levels in the patient with multiple myeloma and the patient after chemotherapy treatment did not differ significantly from the results for the remaining patients. The levels of Klotho in peritoneal effluent were approximately 15fold lower than in plasma. In 8 patients alpha-Klotho in peritoneal effluent was undetectable. There was a strong positive correlation between the serum and dialysate levels of soluble alpha-Klotho $(\mathrm{Rs}=+0.54, \mathrm{p}=0.0016)$.

Serum alpha-Klotho concentrations were significantly higher in women than in men $(186.9 \pm 90.4 v \mathrm{~s}$. $146.1 \pm 87.8 \mathrm{pg} / \mathrm{ml}, \mathrm{p}=0.027)$ but there was no significant correlation with age. The associations of serum alpha-Klotho protein with parameters of kidney function and phosphate metabolism in patients at the onset of PD treatment are presented in Table 2.

Serum Klotho level strongly negatively correlated with 24-hour diuresis $(\mathrm{Rs}=-0.55, \mathrm{p}=0.0044)$ and a weaker correlation with urinary phosphate clearance was observed $(\mathrm{Rs}=-0.39, \mathrm{p}=0.049)$.

The correlation between soluble Klotho and residual diuresis is presented in Figure 1. Residual diuresis was significantly lower in women than in men $(1475.9 \pm 749.4$ vs. $2294.6 \pm 1123.5 \mathrm{~mL}$, $\mathrm{p}=0.013)$.

There was no correlation between serum alpha-Klotho and serum phosphorus, PTH or FGF-23 concentrations.

There were no significant correlations between dialysate alpha-Klotho and other analysed parameters.

When significant correlations of serum Klotho were analyzed in the subgroups stratified by gender, no associations remained significant in men $(\mathrm{Rs}=-0.16, \mathrm{p}=0.63$ for 24 hour diuresis and $\mathrm{Rs}=-0.23, \mathrm{p}=0.47$ for renal phosphate clearance), but in women $(\mathrm{Rs}=-0.69, \mathrm{p}=0.0093$ and $\mathrm{Rs}=-0.04, \mathrm{p}=0.91$, respectively) the negative correlation with 24 hour diuresis became even stronger.

\section{DISCUSSION}

Since the introduction of the sandwich ELISA method for soluble alpha-Klotho assessment, Klotho protein level has been examined in different populations with CKD. However, the data is still scarce and the results might be confusing (Devaraj et al., 2012; Shimamura et al., 2012; Sugiura et al., 2011) probably due to imprecisions of the assay or biological varia- 
Table 2. Associations of serum alpha-Klotho protein with parameters of kidney function and phosphate metabolism in patients at onset of PD/Spearman Rs values are presented.

\begin{tabular}{lc}
\hline Correlated parameters & Serum alpha-Klotho \\
\hline 24-hour diuresis & $-0.55^{* *}$ \\
Serum creatinine & +0.08 \\
Renal creatinine clearance (RRF) & -0.13 \\
Renal phosphate clearance & $-0.40^{*}$ \\
Peritoneal creatinine clearance & +0.18 \\
Peritoneal phosphate clearance & -0.02 \\
Serum P & +0.15 \\
Serum PTH & +0.05 \\
Serum FGF-23 & +0.18 \\
Dialysate alpha-Klotho & $+0.54^{* *}$ \\
\hline
\end{tabular}

${ }^{*} \mathrm{p}<0.05,{ }^{* *} \mathrm{p}<0.01$

tions of Klotho protein. In the Sugiura study (Sugiura et al., 2011) on 30 subjects with CKD (mean serum creatinine $1.63 \pm 1.35 \mathrm{mg} / \mathrm{dL}$ ) the mean values for soluble alpha-Klotho were $1413 \pm 614 \mathrm{pg} / \mathrm{ml}$, while in the control group of healthy adult volunteers the Klotho level was $404 \pm 87 \mathrm{pg} / \mathrm{ml}$. The authors found no correlation between serum Klotho and FGF-23 level. In the Devaraj study (Devaraj et al., 2012) patients with serum creatinine $>2 \mathrm{mg} / \mathrm{dL}$ had significantly higher plasma Klotho level compared to a group with serum creatinine below 1.2 $\mathrm{mg} / \mathrm{dL}$. In another study (Shimamura et al., 2012) on a larger group of patients $(n=292)$ the authors found that soluble Klotho levels were significantly decreased in stage 2 CKD $(616.1 \pm 256.4 \mathrm{pg} / \mathrm{ml})$ compared to stage $1(1442.1 \pm 1410.1 \mathrm{pg} / \mathrm{ml})$, suggesting that alpha-Klotho could represent a new biomarker for the diagnosis of $\mathrm{CKD}$, especially in the early stage. Klotho levels in stages 3-5 CKD were also significantly lower compared to stage 1. Shimamura and co-workers found a positive correlation between RRF and serum soluble Klotho level (Shimamura et al., 2012). It is worthy of a note that none of the patients in stage $5 \mathrm{CKD}$ received renal replacement therapy.

In a study on 53 hemodialysis patients (Yokoyama et al., 2012) the mean level of soluble Klotho was $430 \mathrm{pg} /$ $\mathrm{ml}$, and showed a positive correlation with serum phosphorus $(\mathrm{Rs}=+0.33, \mathrm{p}=0.01)$.

The authors found no significant correlation between Klotho and age, PTH or FGF-23.

Akimoto et al. (2012) performed a study in 36 PD patients and measured serum soluble Klotho concentra-

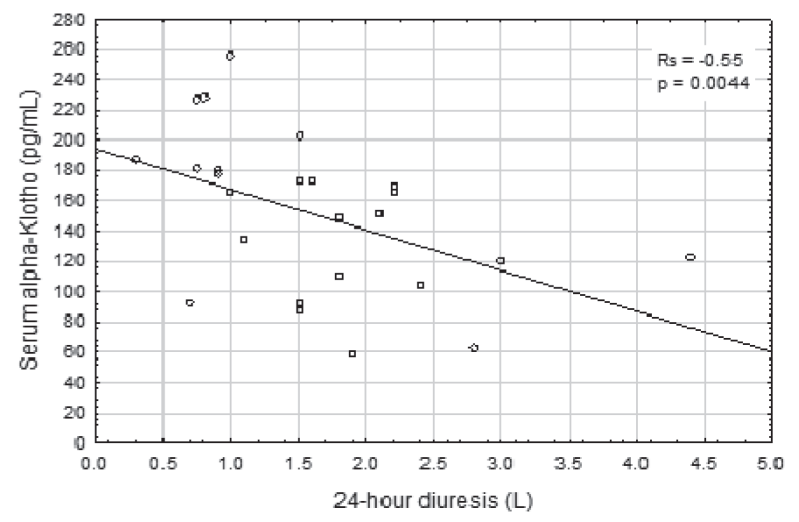

Figure 1. Correlation between serum soluble Klotho level and residual diuresis in incident peritoneal dialysis patients. tion as well as urinary Klotho excretion. Serum soluble Klotho concentration ranged from 194.4 to $990.4 \mathrm{pg} / \mathrm{ml}$ (mean $553.7 \pm 210.4 \mathrm{pg} / \mathrm{ml}$ ). The authors found no apparent correlation between soluble Klotho and creatinine clearance, serum phosphate or calcium. Urinary Klotho excretion, however, was significantly positively correlated with residual renal function.

On the other hand, in a large, population-based study (InCHIANTI), in which approximately one-quarter of the participants had CKD, the authors found no significant relationship between plasma Klotho concentrations and eGFR (Semba et al., 2011). In the study of Seiler et al. (2013) in a cohort of 312 patients with stage 2-4 CKD the levels of serum soluble Klotho were also not associated with GFR.

In our study the mean level of soluble Klotho was $165.9 \pm 90.2 \mathrm{pg} / \mathrm{ml}$. It has to be underlined that, compared to the Akimoto et al's study, the examined group of patients was different: it consisted of incident PD patients with a better RRF and higher urine output (mean $2041 \pm 1067 \mathrm{~mL} /$ day). In our study the serum Klotho concentration was strongly negatively correlated with urine output $(\mathrm{Rs}=-0.55, \mathrm{p}=0.004)$. As secreted Klotho is derived by shedding of the extracellular portion of Klotho from distal tubules we speculate that its lower serum level may be associated with a higher cleavage of Klotho into urine in patients with higher residual diuresis.

Recently, Hu et al. (2010) described a direct, FGF-23 independent, phosphaturic effect of Klotho in mice. In this mode of secreted Klotho action, Klotho protein has to gain access to the lumen of the proximal tubule and then regulates phosphate excretion via the sodium-phosphate transporter NaPi-2a. It seems that Klotho is too large to get to the lumen of the proximal tubule through glomerular filtration. Micropuncture of Bowman's space failed to detect the Klotho antigen. The appearance of Klotho in the proximal lumen could be due to the cleavage and then transcytosis of plasma- or distal tubule-derived Klotho.

The limitation of this study is that we cannot provide data on urinary Klotho excretion, however, Akimoto et al. (2012) showed that the amount of urinary-excreted Klotho was strongly positively associated with the 24-h urine volume $(\mathrm{R}=0.614, \mathrm{p}=0.00114)$.

We found no correlation between RRF and serum soluble Klotho, similarly to those authors. Bearing in mind the data above, we suggest that in incident patients starting peritoneal dialysis lower serum levels of Klotho need not necessarily indicate a risk of poorer prognosis associated with deterioration of kidney function, as they may also partially result from high urinary excretion associated with high 24-hour diuresis.

Except for 8 patients, alpha-Klotho was also detectable in peritoneal effluent, but at much lower concentrations compared with plasma levels. This may be associated with the high molecular mass of this protein. Of note is that there was a significant positive correlation between the plasma and dialysate concentrations of Klotho. In the available literature we found no data on soluble alpha-Klotho concentrations in the effluent of peritoneal dialysis patients. In the Akimoto et al's study the amount of $24-\mathrm{h}$ peritoneal excreted soluble Klotho was correlated with the 24-h peritoneal excreted total protein and albumin (Akimoto et al., 2012).

There was no correlation between serum alpha-Klotho and serum phosphorus concentration, serum PTH or FGF-23. The negative correlation between serum soluble Klotho level and renal phosphate clearance was of bor- 
derline significance $(\mathrm{R}=-0.40, \mathrm{p}=0.049)$. The limitation of our study is the small group of patients, however, the phosphaturic action of soluble Klotho in the proximal tubule proposed by $\mathrm{Hu}$ could be the reason of the observed correlation between renal phosphate clearance and serum Klotho level.

When the group of patients was divided by gender, the significant correlation of serum soluble Klotho with 24-hour diuresis was stronger in women and disappeared in the male subgroup, while the correlation with renal phosphate clearance disappeared in both subgroups. It is of note, however, that residual diuresis was significantly lower in women, which may explain this observation. Nevertheless, the differences between sexes have to be considered with care, as the numbers of patients in the subgroups were small.

Overall, except for the weak correlation of renal phosphate clearance and Klotho, the serum Klotho level had no apparent associations with other parameters of phosphate metabolism and cannot be regarded as a potential marker of calcium-phosphate metabolism disorders in incident PD patients. In the Sugiura et al's study on CKD patients the serum soluble Klotho levels were not correlated with FGF-23 level (Sugiura et al., 2011). In hemodialysis patients the Klotho level was not correlated with FGF-23 level either (Komaba et al., 2012; Yokoyama et al., 2012). To the best of our knowledge we are the first to report a lack of strong associations of serum Klotho level with parameters of phosphate metabolism in incident PD patients.

\section{CONCLUSIONS}

To the best of our knowledge, this is the first report that the urine output, but not the eGFR level, could be associated with the level of serum soluble Klotho in incident PD patients. Serum Klotho level cannot be regarded as a potential marker for calcium-phosphate metabolism disorders in PD patients.

\section{REFERENCES:}

Akimoto T, Shiizaki K, Sugase T, Watanabe Y, Yoshizawa H, Otani N, Numata A, Takeshima E, Yamazaki T, Miki T, Ito C, Pastor JV, Iwazu Y, Saito O, Muto S, Kuro-o M, Kusano E (2012) The relationship between the soluble Klotho protein and the residual renal function among peritoneal dialysis patients. Clin Exp Nephrol 16: $442-447$.
Cheng CY, Kuro-o M, Razzaque MS (2011) Molecular regulation of phosphate metabolism by Fibroblast Growth Factor-23-Klotho system. Adv Chronic Kidney Dis 18: 91-97.

Devaraj S, Syed B, Chien A, Jialal I (2012) Validation of an immunoassay for soluble Klotho protein. Decreased levels in diabetes and increased levels in chronic kidney disease. Am J Clin Pathol 137: 479-485.

Drueke TB, Prie D (2007) Klotho spins the thread of life-what does Klotho do to the receptors of fibroblast growth factor-23 (FGF23)? Nephrol Dial Transplant 22: 1524-1526.

Hu MC, Kuro-o M, Moe OW (2012) The emerging role of Klotho in clinical nephrology. Nephrol Dial Transplant 27: 2650-2657.

Hu MC, Shi M, Zhang J M, Pastor J, Nakatani T, Lanske B, Razzaque MS, Rosenblatt KP, Baum MG, Kuro-o M, Moe OW (2010) Klotho: a novel phosphaturic substance acting as an autocrine enzyme in the renal proximal tubule. FASEB J 24: 3438-3450.

Huang CL, Moe OW (2011) Klotho: a novel regulator of calcium and phosphorus homeostasis. Eur J Physiol 462: 185-193.

Komaba H, Koizumi M, Tanaka H, Takahashi H, Sawada K, Kakuta T, Fukagawa M (2012) Effects of cinacalcet treatment on serum soluble Klotho levels in haemodialysis patients with secondary hyperparathyroidism. Nephrol Dial Transplant 27: 1967-1969.

Kuro-o M, Matsumura Y, Aizawa H, Kawaguchi H, Suga T, Utsugi T, Ohyama Y, Kurabayashi M, Kaname T, Kume E, Iwasaki H, Iida A, Shiraki-Iida T, Nishikawa S, Nagai R, Nabeshima YI (1997) Mutation of the mouse Klotho gene leads to a syndrome resembling ageing. Nature 390: 45-51.

Martin A, David V, Quarles LD (2012) Regulation and function of the FGF23/Klotho endocrine pathways. Physiol Rev 92: 131-155.

Razzaque MS (2009) FGF23-mediated regulation of systemic phosphate homeostasis: is Klotho an essential player? Am J Physiol Renal Physiol 296: F470-F476.

Seiler S, Wen M, Roth HJ, Fehrenz M, Flügge F, Herath E, Weihrauch A, Fliser D, Heine GH (2013) Plasma Klotho is not related to kidney function and does not predict adverse outcome in patients with chronic kidney disease. Kidney Int 83: 121-128.

Semba RD, Cappola AR, Sun K, Bandinelli S, Dalal M, Crasto C, Guralnik JM, Ferrucci L (2011) Plasma Klotho and cardiovascular disease in adults. IAGS 59: 1596-1601.

Shimamura Y, Hamada K, Inoue K, Ogata K, Ishihara M, Kagawa T, Inoue M, Fujimoto S, Ikebe M, Yuasa K, Yamanaka S, Sugiura T, Terada Y (2012) Serum levels of soluble secreted $\alpha$-Klotho are decreased in the early stages of chronic kidney disease, making it a probable novel biomarker for early diagnosis. Clin Exp Nephrol 16: $722-729$.

Sugiura H, Tsuchiya K, Nitta K (2011) Circulating levels of soluble $\alpha$-Klotho in patients with chronic kidney disease. Clin Exp Nephrol 15: 795-796.

Torres PU, Prie D, Molina-Bletry V, Beck L, Silve C, Friedlander G (2007) Klotho: an antiaging protein involved in mineral and vitamin D metabolism. Kidney Int 71: 730-737.

Wang Y, Sun Z (2009) Current understanding of klotho. Ageing Res Rev 8: 43-51.

Yokoyama KK, Imura AA, Ohkido I, Maruyama Y, Yamazaki Y, Hasegawa H, Urae J, Sekino H, Nabeshima Y, Hosoya T (2012) Serum soluble $\alpha$-Klotho in hemodialysis patients. Clin Nephrol 77: $347-351$. 